\section{Fatores associados à interrupção precoce do aleitamento materno: um estudo de coorte de nascimento em dois municípios do Recôncavo da Bahia, Brasil}

\author{
Factors associated with early breastfeeding cessation: \\ a birth cohort study in two municipalities in the \\ Recôncavo region, Bahia State, Brazil
}

\author{
1 Centro de Ciências da \\ Saúde, Universidade Federal \\ do Recôncavo da Bahia, \\ Santo Antônio de Jesus, \\ Brasil. \\ 2 Instituto de Matemática, \\ Universidade Federal da \\ Bahia, Salvador, Brasil. \\ 3 Escola de Nutrição, \\ Universidade Federal da \\ Bahia, Salvador, Brasil. \\ Correspondência \\ F. Demétrio \\ Centro de Ciências da Saúde, \\ Universidade Federal do \\ Recôncavo da Bahia. \\ Av. Carlos Amaral 1015, \\ Santo Antônio de Jesus, $B A$ \\ 44570-000, Brasil. \\ fdemetrio@ufrb.edu.br
}

\begin{abstract}
This study aimed to identify the median duration of breastfeeding and associated factors in a cohort of 531 infants in two municipalities in the Recôncavo region, Bahia State, Brazil. Breastfeeding duration was estimated by survival analysis and its associations by the Cox multivariate model. Median duration of exclusive breastfeeding, mixed breastfeeding with complementary feeding, and total breastfeeding was $74.73,211.25$, and 432.63 days, respectively. Lack of prenatal care increased the risk of shortening exclusive breastfeeding by $173 \%$ (HR $=2.73$; 95\%CI: 1.89-3.93), of adopting mixed breastfeeding with complementary feeding by 83\% (HR = 1.83; 95\%CI: 1.06-3.16), and of discontinuing breastfeeding entirely by $38 \%$ (HR = 1.38; 95\%CI: 1.06-1.81). Both maternal employment and residence in an urban area increased the risk of early breastfeeding cessation. Expansion of access to prenatal care and the safety network for employed mothers living in urban areas could increase breastfeeding duration in the target region.
\end{abstract}

Breast Feeding; Lactation; Infant Nutrition; Cohort Studies
Franklin Demétrio 1

Elizabete de Jesus Pinto 2

Ana Marlúcia Oliveira Assis 3

\section{Introdução}

Estudos produzidos em todo o mundo enfatizam a adequada disponibilidade de energia e nutrientes do leite materno à imaturidade fisiológica do lactente. Destacam-se ainda os fatores de proteção contra as doenças conferidos pelo leite materno, os quais o tornam alimento ideal, capaz de satisfazer as necessidades nutricionais, em especial nos dois primeiros anos de vida. Desse modo, o aleitamento materno promove o crescimento e o desenvolvimento pleno das crianças 1,2,3,4,5,6,7.

As evidências científicas mostram, ainda, que crianças em regime de aleitamento materno exclusivo nos primeiros seis meses, complementado, a partir daí, até pelo menos os dois anos de vida, têm menores chances de desenvolverem doenças crônicas não transmissíveis na infância, adolescência e vida adulta 5 .

Compreende-se, assim, que, por um lado, é consistente o conhecimento sobre os benefícios da amamentação para a saúde da criança; por outro, persistem lacunas importantes sobre os fatores que contribuem para a baixa duração desta prática em várias partes do mundo, inclusive no Brasil 2,3,8.

Recentemente, o Ministério da Saúde 3 identificou aumento na duração mediana de aleitamento materno exclusivo de 23,4 dias em 1999 para 54,1 dias em 2008, para as crianças brasileiras. Tendência similar também foi encontrada para a duração mediana do aleitamento materno total, 
aumentando de 210 dias em 1999 para 341,6 dias em 2008. Salienta-se, ainda, que, embora existam dados recentes que estão permitindo construir a tendência da duração da amamentação no Brasil, estes estudos não disponibilizam informações sobre os fatores que diminuem a duração do aleitamento materno em suas diferentes modalidades para o Brasil, tampouco para áreas ou regiões isoladas do país. Portanto, a identificação destes fatores em pesquisas nacionais e por regiões/áreas pode ser útil para o planejamento e tomada de decisão no campo da saúde e nutrição na infância.

Diante do exposto, o presente estudo tem por objetivo estimar a duração mediana do aleitamento materno nos dois primeiros anos de vida da criança e identificar os fatores associados à interrupção precoce do aleitamento materno em uma coorte de nascimento em dois municípios do Recôncavo da Bahia.

\section{Métodos}

Trata-se de estudo de coorte de nascimento, vinculado a um projeto maior, intitulado $A M A C O M P$ : Amamentação e Alimentação Complementar no Desmame - Estado de Saúde e Nutrição nos Dois Primeiros Anos de Vida - Um Estudo de Coorte, financiado pelo Conselho Nacional de Desenvolvimento Científico e Tecnológico (CNPq), processo nº 505971/04-6, pela Fundação de Amparo à Pesquisa do Estado da Bahia (FAPESB) e pelo Centro Colaborador em Alimentação e Nutrição Região Nordeste II/Ministério da Saúde.

A pesquisa foi realizada nos municípios de Laje e Mutuípe, os quais estão localizados, respectivamente, a $220 \mathrm{~km}$ e $235 \mathrm{~km}$ de Salvador, na região do Recôncavo Sul do Estado da Bahia. O número de habitantes varia de 20.462, em Mutuípe, a 21.108, em Laje, estando domiciliados na área rural, respectivamente, 11.478 (56\%) e 15.076 (71,4\%) (Instituto Brasileiro de Geografia e Estatística.Cidades@.http://www.ibge.gov.br/ cidadesat/topwindow.htm?1, acessado em 31/ Ago/2007). Ambos os municípios foram escolhidos por apresentarem características socioeconômicas equivalentes, com Índice de Desenvolvimento Humano (IDH) similares: 0,53, para Mutuípe, e 0,42, para Laje; apresentam, também, traços ambientais semelhantes aos de outros municípios de pequeno porte do interior do Estado da Bahia. Além disso, considerou-se o fato de haver, previamente, nestes municípios, uma logística de pesquisa já estruturada pelo grupo.

A amostra deste estudo é composta por 531 crianças cujos nascimentos ocorreram entre março de 2005 a outubro de 2006 nas maternida- des públicas dos municípios. As crianças constituíram uma coorte dinâmica, acompanhada por um período de dois anos, com finalização do acompanhamento em outubro de 2008. Ao final do seguimento, as informações sobre aleitamento materno estavam disponíveis para 531 mães e seus filhos.

Esta amostra tem poder (1-b) de 93,5\%, 99\% e $83,5 \%$ de detectar, respectivamente, as medianas de duração do aleitamento materno exclusivo, total e misto complementado. O poder da amostra foi calculado a posteriori, baseando-se nas informações produzidas na própria matriz de dados deste estudo e levou em consideração, respectivamente, a duração média do aleitamento materno exclusivo no primeiro mês ( $\bar{x}=22,96 ; \mathrm{DP}=11,21$ ) e no sexto mês $(\bar{x}=77,37$; $\mathrm{DP}=65,31)$; do aleitamento total no 12o mês ( $\bar{x}=278,0 ; \mathrm{DP}=13,21$ ) e no 24 o mês $(\bar{x}=419,53$; $\mathrm{DP}=248,25)$; e a duração média do aleitamento misto, de 113,36 dias $(\mathrm{DP}=79,4)$ para o sexto mês e de 244,48 (DP = 220,55) para o 24o mês. Os cálculos levaram em consideração o nível de significância (a) de $5 \%$ para testes bicaudais 9 .

Ainda na maternidade, as mães foram informadas dos objetivos do estudo e convidadas a participar. Aquelas que concordaram assinaram o Termo de Consentimento Livre e Esclarecido; a analfabeta o fez por meio da impressão digital. Esse consentimento foi uma condição para participação da mãe e da criança na pesquisa. Foram excluídos do estudo: nascimentos gemelares; crianças portadoras de defeitos congênitos da cavidade oral, os quais impedissem a amamentação, ou de outras condições que impedissem a aferição das medidas antropométricas; recémnascidos de mães portadoras de HIV.

Neste estudo, o desfecho foi definido como o tempo decorrido do nascimento até a finalização da duração do aleitamento materno (falha). Adotou-se a escala de medida de tempo em dias. Desse modo, as variáveis do desfecho foram representadas pelas seguintes condições: (i) aleitamento materno exclusivo, caracterizado pelo oferecimento exclusivo de leite materno à criança, não sendo permitido o consumo nem mesmo de água 10; situação diferente desta definição indicava o término do evento. (ii) Aleitamento misto complementado, caracterizado pelo consumo do leite de vaca, associado ao leite materno, e pela inclusão de outros alimentos ao esquema alimentar. Esse regime alimentar não encontra definição dentre aqueles nomeados pela Organização Mundial da Saúde (OMS) 10, mas foi considerado neste estudo por ser adotado com muita frequência em várias regiões do Nordeste do Brasil, associando-se ao declínio dos níveis de hemoglobina em crianças menores de 24 meses 
de idade, em comparação com o regime de aleitamento materno complementado 11. Quando o leite materno era excluído do esquema alimentar da criança, o evento era dado por encerrado. (iii) Aleitamento materno total, entendido pelo consumo do leite materno em qualquer regime 10; quando este era excluído do cardápio da criança, o evento era dado por terminado.

Em estudo de seguimento, é possível captar as mudanças processadas no regime alimentar da criança com todo o dinamismo que este evento apresenta. Assim, uma criança pode ter efetuado mudança no regime alimentar no momento do contato com o serviço de saúde e depois voltar para o regime anterior, até mesmo como resultado do aconselhamento. Esta condição ocorreu entre algumas das crianças do presente estudo. Logo, decidiu-se por considerar como regime desse período aquele em que a criança se encontrava antes, caso esta mudança tenha se dado há menos de trinta dias do novo contato. Se o tempo sob o novo regime excedeu trinta dias, este foi adotado como o praticado no período em questão.

Um conjunto de características antropométricas, biológico-obstétricas, sociodemográficas e ambientais maternas e das crianças, além de características de consumo alimentar nos dois primeiros anos de vida, compuseram as variáveis independentes deste estudo. Essas variáveis são representadas por condições sociodemográficas (escolaridade materna, trabalho materno fora do domicílio depois do parto); as variáveis biológicas da mãe e da criança são integradas pela idade materna, sexo da criança, cor da pele e altura maternas; as variáveis obstétricas e antropométricas são compostas pela realização de consulta prénatal, tipo de parto, tempo de gestação, peso ao nascer, peso pré e pós-gestacional; a variável ambiental é composta pela área de residência e pelo índice de condições de moradia. A categorização dessas variáveis está disposta de forma autoexplicativa na Tabela 1 .

A produção de dados sobre as características sociodemográficas, ambientais e biológicoobstétricas maternas, ocorreu no primeiro mês de vida da criança na visita domiciliar. Informações sobre o consumo alimentar do primeiro até o sexto mês de vida da criança foram coletadas mensalmente e, a partir daí até os 24 meses de vida, as coletas foram semestrais. No primeiro mês, a coleta foi realizada no domicílio; nos demais períodos, ocorreu durante a consulta na unidade básica de saúde (UBS) dos municípios. Adotouse o método recordatório de 24 horas para coletar informações, registradas em questionários padronizados, sobre o consumo alimentar das crianças; foram acrescidos dados sobre o ofereci- mento de alimentos complementares, ocorrido entre a consulta anterior e a em curso. Quando, por qualquer motivo, a mãe não comparecia à consulta, a equipe do projeto fazia a coleta das informações no domicílio da criança.

O peso ao nascer foi obtido na sala de parto pela equipe de obstetrícia da maternidade, utilizando-se balança microeletrônica da marca Filizola, modelo E-150/3P, com precisão de $10 \mathrm{~g}$ (Filizola, São Paulo, Brasil). O comprimento ao nascer foi aferido pela equipe de entrevistadores, utilizando-se infantômetro de madeira com fita antropométrica inelástica, instrumento já testado em outros estudos do grupo.

O peso pós-parto e a altura materna foram obtidos na maternidade, no momento da alta hospitalar, quando a mãe já se encontrava hemodinamicamente estável. O peso foi aferido em balança eletrônica, marca Filizola, modelo E-150/3P, com capacidade para $150 \mathrm{~kg}$ e precisão de 100g; para a medição da altura, utilizou-se estadiômetro (Leicester Height Measure, Londres, Inglaterra), com a leitura realizada no milímetro mais próximo. O peso pré-gravídico foi referido pela puérpera. Procederam-se às aferições conforme as técnicas descritas por Lohman et al. 12 . Para a classificação do estado antropométrico materno pré e pós-gestacional, adotaram-se os pontos de corte de índice de massa corporal da OMS.

As condições de moradia foram avaliadas por meio do índice ambiental, o qual foi construído a partir de oito situações indicativas das condições físicas e ambientais do domicílio e de seu entorno, adaptado de Oliveira et al. 13 .

Em atenção ao pressuposto ético, no primeiro contato na maternidade, foram distribuídas às mães cartilhas contendo informações sobre a alimentação saudável nos dois primeiros anos de vida da criança e sobre a alimentação da lactante, segundo o preconizado pelo Ministério da Saúde 14 . A cada contato entre a mãe e a equipe de projeto, reforçava-se a importância da alimentação saudável nos dois primeiros anos de vida e no período da lactância. Quando a criança e/ou a lactante apresentavam algum problema de saúde, a equipe os encaminhava para atendimento nos serviços de saúde dos municípios.

Adotou-se a proporção para caracterizar a população do estudo. As curvas da duração do aleitamento materno foram estimadas pela análise de sobrevivência, utilizando-se o método não paramétrico de Kaplan \& Meier 15. A associação entre as variáveis de exposição e a duração do aleitamento materno foi avaliada por meio da regressão semiparamétrica de Cox e interpretada pela razão de risco (hazard ratio - HR), com intervalo de 95\% de confiança (IC95\%). 
Caracterização sociodemográfica, ambiental, biológica, obstétrica e antropométrica das mães e suas crianças. Laje/Mutuípe Bahia, Brasil, 2005-2008.

\begin{tabular}{|c|c|c|}
\hline Variável & n & $\%$ \\
\hline \multicolumn{3}{|c|}{ Escolaridade materna (anos de estudo) [ $n=527]$} \\
\hline $8 \mathrm{ou}+$ & 127 & 24,2 \\
\hline Até 7 & 397 & 75,8 \\
\hline \multicolumn{3}{|c|}{ Trabalho fora do domicílio pós-parto $[n=452]^{*}$} \\
\hline Não & 305 & 67,5 \\
\hline $\operatorname{Sim}$ & 147 & 32,5 \\
\hline \multicolumn{3}{|l|}{ Idade materna (anos) $[n=516]$ * } \\
\hline$\geq 20$ & 355 & 68,8 \\
\hline$<20$ & 161 & 31,2 \\
\hline \multicolumn{3}{|l|}{ Sexo da criança $[n=527]$} \\
\hline Masculino & 275 & 52,2 \\
\hline Feminino & 252 & 47,8 \\
\hline \multicolumn{3}{|c|}{ Índice de condições de moradia $[n=525]$ * } \\
\hline Adequado & 188 & 35,8 \\
\hline Inadequado & 337 & 64,2 \\
\hline \multicolumn{3}{|l|}{ Área de residência $[n=526]$ * } \\
\hline Rural & 369 & 70,2 \\
\hline Urbana & 157 & 29,8 \\
\hline \multicolumn{3}{|l|}{ Cor de pele $[n=489]$ * } \\
\hline Não preta & 377 & 77,1 \\
\hline Preta & 112 & 22,9 \\
\hline \multicolumn{3}{|c|}{ Realização de pré-natal [n = 527] } \\
\hline $\operatorname{Sim}$ & 366 & 69,4 \\
\hline Não & 161 & 30,6 \\
\hline \multicolumn{3}{|l|}{ Tipo de parto $[n=525]$ * } \\
\hline Vaginal & 402 & 76,6 \\
\hline Cesárea & 123 & 23,4 \\
\hline \multicolumn{3}{|l|}{ Tempo de gestação $[n=447]$ * } \\
\hline Recém-nascido pré-termo & 54 & 12,1 \\
\hline Recém-nascido a termo & 393 & 87,9 \\
\hline \multicolumn{3}{|l|}{ Peso ao nascer (g) $[n=526]$ * } \\
\hline$\geq 3.000$ & 355 & 67,5 \\
\hline$<3.000$ & 171 & 32,5 \\
\hline \multicolumn{3}{|l|}{ Altura materna $(\mathrm{cm})[\mathrm{n}=522]$ * } \\
\hline$\geq 150$ & 467 & 89,5 \\
\hline$<150$ & 55 & 10,5 \\
\hline \multicolumn{3}{|c|}{ Estado antropométrico materno pré-gestacional $[n=403]$ * } \\
\hline Sem excesso de peso & 332 & 82,4 \\
\hline Excesso de peso & 71 & 17,6 \\
\hline \multicolumn{3}{|c|}{ Estado antropométrico materno pós-parto $[n=501]$ * } \\
\hline Sem excesso de peso & 343 & 68,5 \\
\hline Excesso de peso & 158 & 31,5 \\
\hline
\end{tabular}

* Dados faltantes. 
Para a aplicação desta técnica estatística, primeiramente, interpretou-se o comportamento da variável resposta ao fim da exposição no tempo, como segue: (i) para cada criança, foi caracterizada a situação da sobrevivência, também denominada de falha, interpretada pelo tempo decorrido entre o nascimento até a ocorrência do evento de interesse; (ii) para cada criança, foi definida a situação de censura, interpretada quando o evento de interesse não havia ocorrido até o final da observação, ou pela perda da criança durante o acompanhamento.

A seguir foram avaliados os pressupostos para aplicação da técnica da regressão de Cox. A suposição de riscos proporcionais foi atendida, indicando que esse modelo de regressão era adequado aos dados deste estudo. Para essa finalidade, foram adotados o método gráfico descritivo e valor de $\mathrm{p}<0,05$ para rejeição da hipótese de que os riscos são proporcionais 16 . A situação extrema de violação desse pressuposto caracteriza-se por curvas que se cruzam. E, finalmente, para identificar os fatores associados à duração dos diferentes tipos de aleitamento materno, foi utilizado o teste log rank proposto por Mantel 17. Nesse estágio, as variáveis cujas associações mostraram o valor de $\mathrm{p}<0,20$ e atenderam também ao critério da proporcionalidade do risco foram selecionadas para integrarem o modelo de regressão multivariado de Cox. Foram ajustados três modelos de regressão multivariados de Cox, um para cada modalidade de aleitamento investigada.

Para a construção do banco de dados e análises estatísticas, utilizaram-se, respectivamente, os softwares Epi Info versão 6.04 (Centers for Disease Control and Prevention, Atlanta, Estados Unidos) e Stata versão 10.0 (Stata Corp., College Station, Estados Unidos).

Este estudo obteve a aprovação do Comitê de Ética em Pesquisa da Maternidade Climério de Oliveira, da Universidade Federal da Bahia (parecer $n^{\circ}$. 74/2005).

\section{Resultados}

A caracterização socioambiental, demográfica, biológico-obstétrica e antropométrica materna e das crianças está apresentada na Tabela 1. A escolaridade menor do que oito anos de estudo foi identificada em $75,8 \%$ das mães. A condição de "não trabalhar fora do domicílio após o parto" foi referida por $67,5 \%$ delas; a idade de $\geq 20$ anos foi a mais frequente entre as mães (68,8\%), e a cor da pele branca/parda (não preta) foi referida por $77,1 \%$ delas. As condições inadequadas de moradia foram identificadas para $64,2 \%$ das mães e suas crianças, e 70,2\% das residências estavam localizadas na área rural. O pré-natal foi realizado por $69,4 \%$ das mães, e o baixo peso/eutrofia (sem excesso de peso) ao início da gestação e no período pós-parto foi, respectivamente, de $82,4 \%$ e $68,5 \%$ entre elas.

O parto vaginal foi a forma de nascimento de $76,6 \%$ das crianças, e o sexo masculino foi registrado para $52,2 \%$ dos recém-nascidos. O peso adequado ao nascer $(\geq 2.500 \mathrm{~g})$ foi identificado para $67,5 \%$ das crianças, e $87,9 \%$ nasceram a termo. Nenhum caso que atendesse aos critérios de exclusão foi observado neste estudo.

Observou-se que 98,1\% das crianças iniciaram a amamentação ao peito. A duração mediana de aleitamento materno exclusivo foi de 74,73 dias. Para o aleitamento misto complementado, esta duração foi de 211,25 dias e, para o aleitamento total, de 432,63 dias (dados não apresentados em tabela). Aleitamento materno predominante, aleitamento materno complementado e aleitamento misto tiveram, respectivamente, curta duração mediana de 11,3, 9,4 e 12,0 dias; por isso, essas categorias do regime alimentar não foram incluídas neste estudo.

Os resultados das curvas de sobrevivência estimadas pelo método de Kaplan-Meier para os diferentes tipos de aleitamento, segundo variáveis selecionadas, indicaram que a duração mediana do aleitamento materno exclusivo até os seis meses de idade é proporcionalmente menor entre as crianças cujas mães não realizaram o pré-natal e residiam na zona urbana, em comparação com aquelas cujas mães fizeram o pré-natal e viviam na área rural (Figuras la e 1b). A duração do regime de aleitamento misto complementado foi proporcionalmente maior entre as crianças cujas mães residiam na zona urbana, não realizaram o pré-natal e trabalharam fora do domicílio após o parto, comparativamente àquelas cujas mães residiam na zona rural, realizaram o pré-natal e não trabalharam fora do domicílio após o parto (Figuras 1c, 1d e 1e). Para o aleitamento materno total, observou-se que sua duração por um período de 720 dias (24 meses) foi proporcionalmente menor para as crianças cujas mães residiam na zona urbana e não realizaram o pré-natal, em comparação àquelas cujas mães eram da zona rural e realizaram o pré-natal (Figuras lf e 1g).

Os resultados do teste log rank para identificar as variáveis preditoras do desfecho estão apresentados na Tabela 2. Para o aleitamento materno exclusivo, as variáveis área de residência e realização de pré-natal foram selecionadas a fim de compor o modelo multivariado de Cox. Para os regimes de aleitamento misto complementado e materno total, foram selecionadas as variáveis área de residência, trabalho materno fora do domicílio depois do parto e realização 
Figura 1

Curvas de sobrevivência S(t), estimadas pelo método de Kaplan-Meier, para o tempo de aleitamento materno exclusivo, segundo realização de pré-natal (1a) e área de residência (1b); para o tempo de aleitamento misto complementado, segundo área de residência (1c), realização de pré-natal (1d) e trabalho materno fora do domicílio pós-parto (1e); para o tempo de aleitamento materno total, segundo área de residência (1f) e realização de pré-natal (1g). Laje/Mutuípe, Bahia, Brasil, 2005-2008.

1a) Aleitamento materno exclusivo e realização de pré-natal.

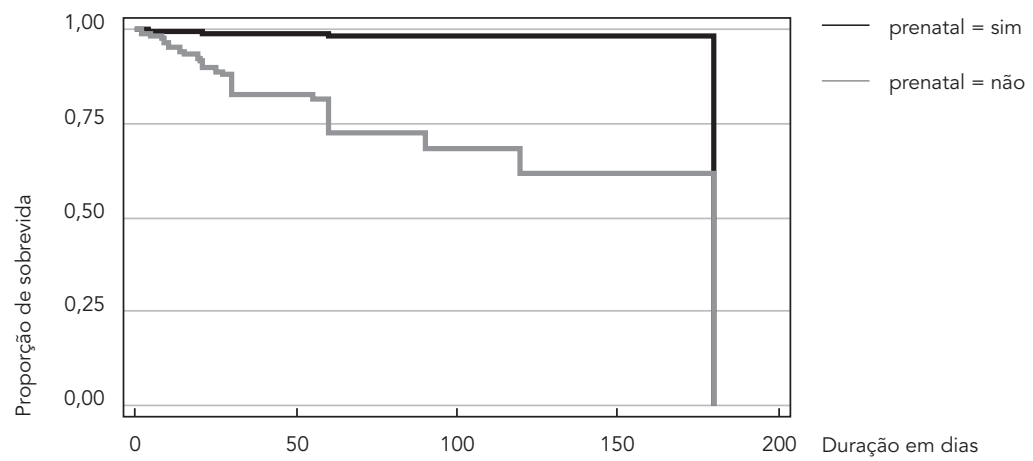

1b) Aleitamento materno exclusivo e área de residência.

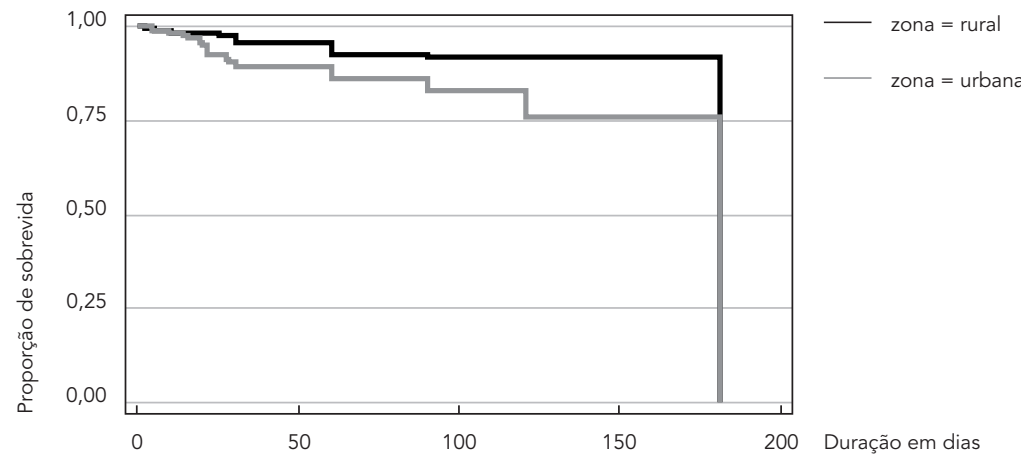

1c) Aleitamento misto complementado e área de residência.

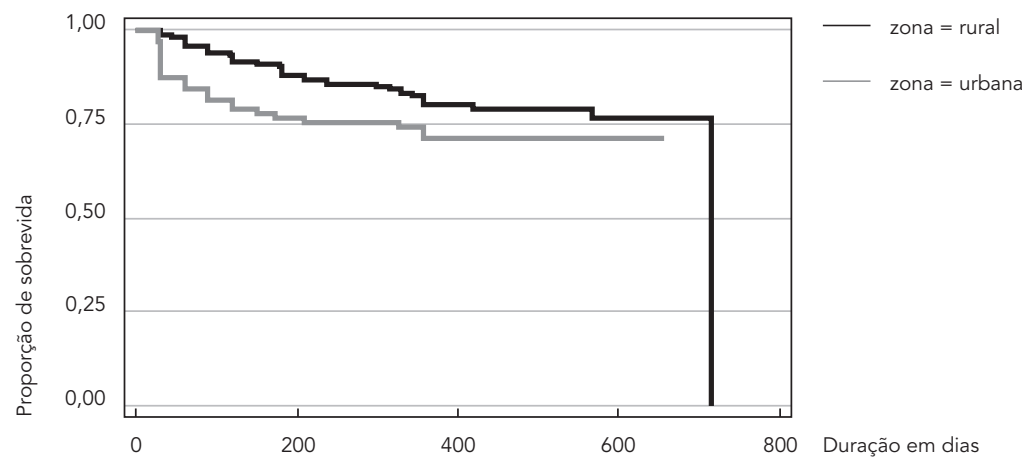


1d) Aleitamento misto complementado e realização de pré-natal.

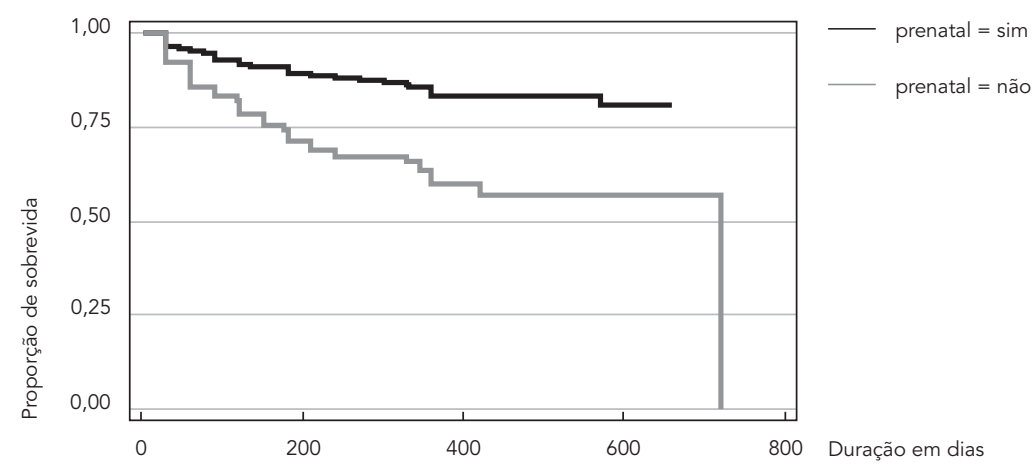

1e) Aleitamento misto complementado e trabalho materno fora do domicílio pós-parto.

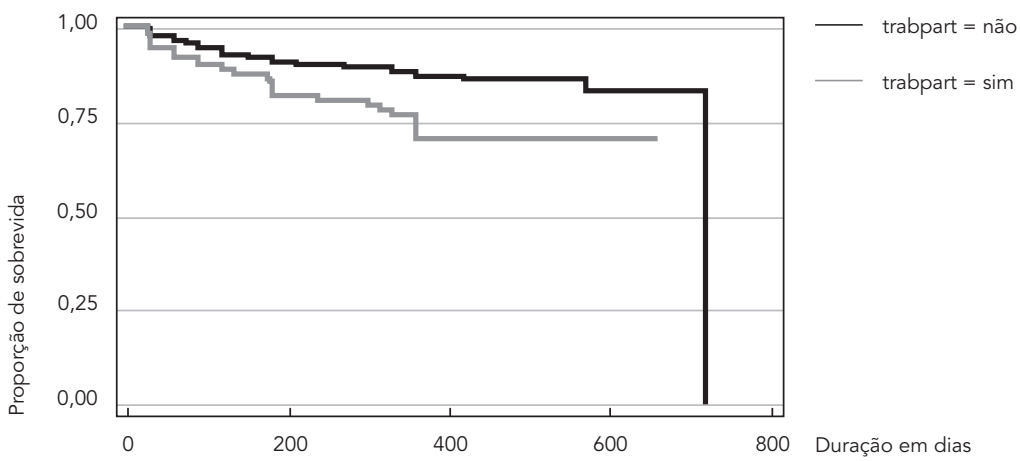

1f) Aleitamento materno total e área de residência.

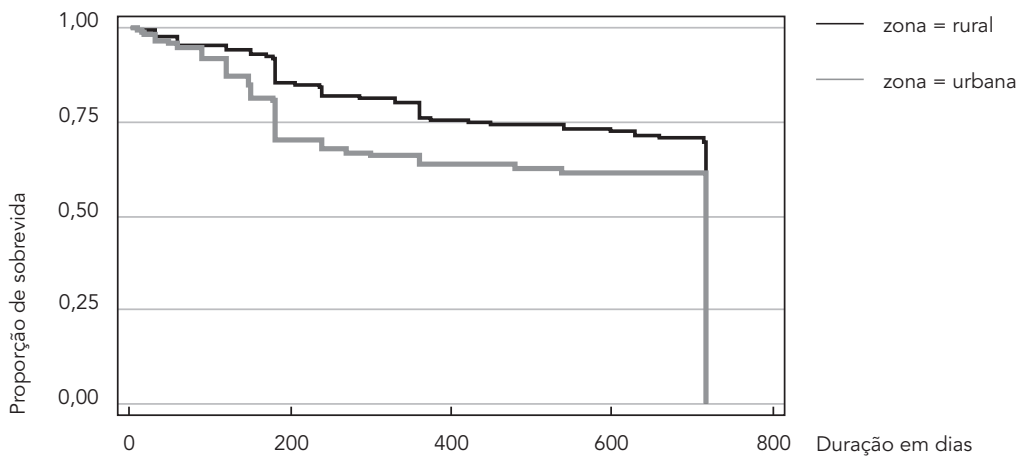


1g) Aleitamento materno total e realização de pré-natal.

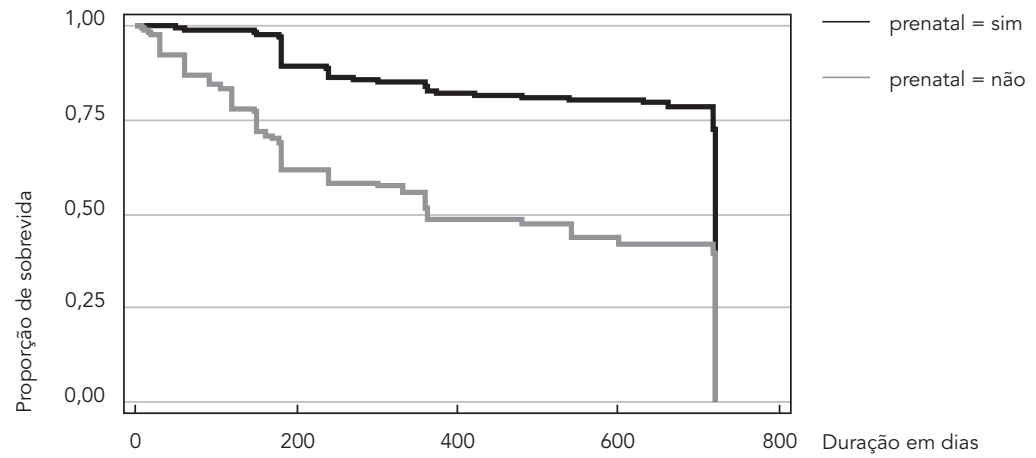

de pré-natal. As variáveis índice de condições de moradia, peso ao nascer e estado antropométrico materno pós-parto, embora tenham atingido o critério do valor de $\mathrm{p}<0,20$, não foram incluídas no modelo multivariado de Cox, pois se observou violação do pressuposto da proporcionalidade dos riscos.

Na Tabela 3, apresentam-se os resultados das análises para as três modalidades de aleitamento estudadas e as variáveis de exposição, obtidas pela modelagem multivariada de Cox. Para o aleitamento materno exclusivo, as variáveis realização de pré-natal e área de residência se associaram significantemente ao evento. Logo, crianças cujas mães não realizaram o pré-natal e residiam na área urbana tiveram 2,73 $(\mathrm{HR}=2,73$; IC95\%: 1,89-3,93) e 1,61 (HR = 1,61; IC95\%: 1,082,40 ), respectivamente, mais chance de interrupção precoce do aleitamento materno exclusivo, quando comparadas às crianças cujas mães realizaram o pré-natal e residiam na área rural.

Em relação ao aleitamento misto complementado, identificou-se que o risco de as crianças receberem, concomitantemente, leite materno, leite de vaca ou similar e outros alimentos foi $116 \%$ mais elevado (HR = 2,16; IC95\%: 1,22-3,82) quando a mãe não realizava o pré-natal, e $83 \%$ mais alto (HR = 1,83; IC95\%: 1,06-3,16) quando a mãe trabalhava fora do domicílio após o parto, em relação àquelas cujas mães, respectivamente, realizaram o pré-natal e não trabalharam fora do domicílio depois do parto.

Para o aleitamento materno total, o resultado da análise multivariada indicou que residir na zona urbana eleva em $38 \%$ o risco de a criança ser desmamada antes dos dois anos de vida $(\mathrm{HR}=$
1,38; IC95\%: 1,06-1,81), quando se compara com o risco de se desmamar a criança da área rural. Finalmente, para as crianças cujas mães não fizeram o pré-natal, o risco do desmame foi $139 \%$ maior (HR = 2,39; IC95\%: 1,83-3,13), quando são comparadas com aquelas cujas mães fizeram o pré-natal.

\section{Discussão}

No presente trabalho, a duração mediana do aleitamento materno exclusivo (74,73 dias) mostrou-se mais elevada do que a nacional, estimada pelo Ministério da Saúde 3,18,19 em 1999 (23,4 dias), em 2006 (42 dias) e em 2008 (53,1 dias). Tendência semelhante foi identificada, neste estudo, para o aleitamento materno total $(423,63$ dias), quando comparada à de 1999 (210 dias) e à de 2009 (341,6 dias), igualmente estimadas pelo Ministério da Saúde 3,18 para o país. Observou-se, ainda, taxa de iniciação do aleitamento materno $(98,1 \%)$ ligeiramente mais elevada do que a encontrada para o Brasil como um todo em 199918 (97\%) e expressivamente maior do que a de $2008{ }^{3}(67,7 \%)$.

Outros estudos de coorte desenvolvidos no Brasil registraram menor duração mediana para os diversos tipos de aleitamento materno do que a identificada neste estudo. Durações medianas de 23 dias para o aleitamento materno exclusivo e de 205 dias para aleitamento materno total foram registradas no Sudeste ${ }^{20}$. Baixa duração do aleitamento materno total foi também observada para crianças acompanhadas no Sul do país (204 dias) 21. No Nordeste do Brasil, registram-se 
Valores de $\mathrm{p}$ * obtidos na análise bivariada do teste log rank, por meio da regressão de Cox, para o tempo de aleitamento materno exclusivo, misto complementado e total, segundo variáveis maternas e da criança. Laje/Mutuípe, Bahia, Brasil, 2005-2008.

\begin{tabular}{|c|c|c|c|}
\hline \multirow[t]{2}{*}{ Variáveis } & \multicolumn{3}{|c|}{ log rank } \\
\hline & $\begin{array}{c}\text { Aleitamento materno } \\
\text { exclusivo }\end{array}$ & $\begin{array}{l}\text { Aleitamento misto } \\
\text { complementado }\end{array}$ & Aleitamento materno total \\
\hline $\begin{array}{l}\text { Escolaridade materna (anos de estudo) } \\
8 \text { ou }+ \\
\text { Até } 7\end{array}$ & 0,887 & 0,379 & 0,916 \\
\hline $\begin{array}{l}\text { Trabalho fora do domicílio pós-parto } \\
\text { Não } \\
\text { Sim }\end{array}$ & 0,970 & 0,005 & 0,060 \\
\hline $\begin{array}{l}\text { Idade materna (anos) } \\
\qquad 20 \\
\quad<20\end{array}$ & 0,429 & 0,326 & 0,343 \\
\hline $\begin{array}{l}\text { Sexo da criança } \\
\text { Masculino } \\
\text { Feminino }\end{array}$ & 0,516 & 0,192 & 0,817 \\
\hline $\begin{array}{l}\text { Índice de condições de moradia } \\
\text { Adequado } \\
\text { Inadequado }\end{array}$ & 0,143 & 0,181 & 0,168 \\
\hline $\begin{array}{l}\text { Área de residência } \\
\text { Rural } \\
\text { Urbana }\end{array}$ & 0,032 & 0,031 & 0,043 \\
\hline $\begin{array}{l}\text { Cor de pele } \\
\text { Não preta } \\
\text { Preta }\end{array}$ & 0,909 & 0,185 & 0,611 \\
\hline $\begin{array}{l}\text { Realização de pré-natal } \\
\text { Sim } \\
\text { Não }\end{array}$ & 0,000 & 0,000 & 0,000 \\
\hline $\begin{array}{l}\text { Tipo de parto } \\
\text { Vaginal } \\
\text { Cesárea }\end{array}$ & 0,969 & 0,859 & 0,705 \\
\hline $\begin{array}{l}\text { Tempo de gestação } \\
\text { Recém-nascido pré-termo } \\
\text { Recém-nascido a termo }\end{array}$ & 0,879 & 0,852 & 0,980 \\
\hline $\begin{array}{l}\text { Peso ao nascer }(\mathrm{g}) \\
\qquad \begin{array}{l}\geq 3.000 \\
<3.000\end{array}\end{array}$ & 0,642 & 0,095 & 0,956 \\
\hline $\begin{array}{l}\text { Altura materna }(\mathrm{cm}) \\
\quad \geq 150 \\
\quad<150\end{array}$ & 0,988 & 0,748 & 0,732 \\
\hline $\begin{array}{l}\text { Estado antropométrico materno pré-gestacional } \\
\text { Sem excesso de peso } \\
\text { Excesso de peso }\end{array}$ & 0,953 & 0,374 & 0,466 \\
\hline $\begin{array}{l}\text { Estado antropométrico materno pós-parto } \\
\text { Sem excesso de peso } \\
\text { Excesso de peso }\end{array}$ & 0,140 & 0,005 & 0,016 \\
\hline
\end{tabular}

* Nível de significância < 0,05. 
Hazard ratio (HR) bruta e ajustada e os respectivos intervalos de confiança, segundo variáveis associadas ao aleitamento materno exclusivo, misto complementado e total, obtidos pela análise do modelo de regressão de Cox. Laje/Mutuípe, Bahia, Brasil, 2005-2008.

\begin{tabular}{|c|c|c|c|c|}
\hline Variáveis & $\mathrm{HR}_{\text {bruta }}$ & $\mathrm{IC} 95 \%$ & HR ajustada & IC95\% \\
\hline \multicolumn{5}{|c|}{ Tempo de aleitamento materno exclusivo } \\
\hline \multicolumn{5}{|c|}{ Área de residência } \\
\hline Rural & 1,00 & & 1,00 & \\
\hline Urbana & 1,53 & $1,03-2,28$ & 1,61 & $1,08-2,40$ \\
\hline \multicolumn{5}{|c|}{ Realização de pré-natal } \\
\hline Sim & 1,00 & & 1,00 & \\
\hline Não & 2,67 & $1,85-3,83$ & 2,73 & $1,89-3,93$ \\
\hline \multicolumn{5}{|c|}{ Trabalho materno fora do domicílio pós-parto } \\
\hline \multicolumn{5}{|l|}{ Não } \\
\hline Sim & NS & NS & & \\
\hline \multicolumn{5}{|c|}{ Tempo de aleitamento misto complementado } \\
\hline \multicolumn{5}{|c|}{ Área de residência } \\
\hline Rural & 1,00 & & 1,00 & \\
\hline Urbana & 1,68 & $1,04-2,71$ & 1,75 & $1,01-3,06$ \\
\hline \multicolumn{5}{|c|}{ Realização de pré-natal } \\
\hline Sim & 1,00 & & 1,00 & \\
\hline Não & 2,80 & $1,76-4,45$ & 2,16 & $1,22-3,82$ \\
\hline \multicolumn{5}{|c|}{ Trabalho materno fora do domicílio pós-parto } \\
\hline Não & 1,00 & & 1,00 & \\
\hline Sim & 2,13 & $1,25-3,62$ & 1,83 & $1,06-3,16$ \\
\hline \multicolumn{5}{|c|}{ Tempo de aleitamento total } \\
\hline \multicolumn{5}{|c|}{ Área de residência } \\
\hline Rural & 1,00 & & 1,00 & \\
\hline Urbana & 1,32 & $1,00-1,72$ & 1,38 & $1,06-1,81$ \\
\hline \multicolumn{5}{|c|}{ Realização de pré-natal } \\
\hline Sim & 1,00 & & 1,00 & \\
\hline Não & 2,36 & $1,81-3,08$ & 2,39 & $1,83-3,13$ \\
\hline \multicolumn{5}{|c|}{ Trabalho materno fora do domicílio pós-parto } \\
\hline Não & NS & NS & & \\
\hline Sim & & & & \\
\hline
\end{tabular}

IC95\%: intervalo de 95\% de confiança; NS: não significante.

medianas de aleitamento materno exclusivo e total, respectivamente, de 0 e 94 dias 22. Essas durações podem ser consideradas as mais baixas já registradas na história da prática do aleitamento materno no país.

Estudos transversais também têm apresentado baixa duração do aleitamento materno exclusivo, variando de 30 a 30,6 dias nas cidades do Rio de Janeiro 23 e de Salvador 8 , respectivamente. Duração menor de trinta dias foi registrada para o Estado de Pernambuco 24.

Nesta pesquisa, $50 \%$ das crianças receberam leite de vaca associado ao leite materno e a outros alimentos por 211,25 dias e antes de comple- tarem dois anos de vida, representando expressivo tempo de exposição ao leite de vaca em idade precoce. A inclusão deste leite no regime alimentar da criança é prejudicial à sua saúde e nutrição antes dos 24 meses de vida 10,11,25,26,27,28.

Foi possível identificar também que $50 \%$ das crianças mamaram ao peito por 432,63 dias durante os seus dois primeiros anos de vida. Além de este tempo ser ainda insatisfatório, soma-se o fato de que metade das crianças nessa idade já não usufruía da proteção do leite materno.

A duração do aleitamento materno total identificada neste estudo é 2,3 vezes maior do que aquela identificada há cinco anos (192 dias) 
para um dos municípios investigados 29 . Esses resultados põem em evidência que a prática do aleitamento materno nesses municípios pode ser considerada exitosa, ainda que não tenha atendido ao preconizado, como o tempo adequado para os regimes de aleitamento materno exclusivo e total. Salienta-se que ambos os municípios não estavam credenciados junto ao Programa Iniciativa Hospital Amigo da Criança e não adotavam quaisquer atividades que promovessem e estimulassem o aleitamento materno até o desenvolvimento desta investigação. Esses resultados positivos podem ter sido influenciados pelo incentivo ao aleitamento materno e pelo aconselhamento para a alimentação saudável nos dois primeiros anos de vida dispensados às mães e suas crianças desta coorte, as quais integravam um projeto maior desenvolvido nos municípios que abrigaram o estudo.

Durante as duas últimas décadas, têm sido observados avanços crescentes no leque de ações do Programa da Atenção Básica do Ministério da Saúde 3 e das parcerias instituídas com organismos internacionais 2 , reestruturando as políticas públicas de promoção e incentivo ao aleitamento materno, o que resulta na melhoria dos indicadores 3,25 deste evento. Entretanto, as unidades básicas de saúde dos dois municípios investigados não adotam estratégias resolutivas para elevar a qualidade desses indicadores 2,10, traduzidas na duração do aleitamento materno exclusivo até os seis meses da criança e complementado, pelo menos, até os dois anos de vida desta. Assim, a meta de estender a duração do aleitamento materno exclusivo até os seis meses de vida da criança ainda permanece como grande desafio para vários países, em especial para o Brasil e suas áreas geográficas 10,30.

Esta dificuldade é respaldada pelos resultados deste estudo, segundo os quais a mediana da duração do aleitamento materno exclusivo foi mais elevada do que aquela registrada recentemente para o Brasil ${ }^{3}$. No entanto, esse aumento ainda se encontra muito aquém do que é recomendado pelo Ministério da Saúde do Brasil e da OMS.

A duração do aleitamento materno pode ser influenciada por diversos fatores, incluindo as condições sociodemográficas, ambientais, biológicas, obstétricas e culturais maternas, além daqueles relacionados à assistência prestada pelos serviços de saúde 3,30,31,32. No presente estudo, a ausência da realização do pré-natal é um fator que explica tanto a interrupção precoce da amamentação exclusiva, quanto a adoção do aleitamento misto complementado. Resultados semelhantes foram registrados por outros estudos 32,33 .
Essas evidências destacam o espaço do prénatal como a porta de entrada para o cuidado primário no campo da saúde e nutrição materno-infantil e decisão da mulher em amamentar seus filhos. Sendo assim, o incentivo ao aleitamento materno no pré-natal é uma ação de baixa complexidade, constituindo-se em importante aliada na promoção da saúde e nutrição materno-infantil 30,34,35.

Destaca-se a importância de dotar os serviços de saúde e os setores sociais com estruturas físicas e logísticas motivadoras do aleitamento materno no âmbito do pré-natal, a fim de ecoar os anseios da mulher à prática da amamentação.

Nesta investigação, o trabalho materno fora do domicílio imprime risco 1,83 vezes maior para o oferecimento precoce de leite de vaca e de outros alimentos diferentes do leite humano à criança. Riscos similares foram identificados por Baptista et al. 35 e Scott et al. 33 para o trabalho materno fora do lar nos seis meses pós-parto. Portanto, é possível especular que a crescente participação da mulher no mercado de trabalho tem aumentado o risco do oferecimento precoce de alimentos diferentes do leite materno, em especial, o leite de vaca, tornando o regime alimentar incompatível com as diretrizes da alimentação saudável nos dois primeiros anos de vida 30,36 .

Esta realidade é documentada tanto nos países desenvolvidos, quanto em municípios de grande e pequeno portes dos países em desenvolvimento 37,38. Estudo realizado recentemente com mulheres trabalhadoras norte-americanas revelou que estratégias de ampliação de espaços para a prática da amamentação no ambiente de trabalho contribuíram significantemente para a maior duração do aleitamento materno ${ }^{39}$. Assim, tem sido fortalecida a estratégia da rede social de apoio ao aleitamento materno, em especial entre as mães trabalhadoras, por constituírem grupo de maior risco de oferecer precocemente alimentos diferentes do leite materno à criança $30,37,38,39,40$.

Para o Brasil, a recomendação da OMS referente à duração da amamentação exclusiva de seis meses é amparada pela nova legislação trabalhista que torna facultativa, para as empresas, a extensão da licença-maternidade de 120 para 180 dias, conforme Lei $n^{\circ}$. 11.770/08 41. No entanto, um expressivo contingente de mulheres trabalhadoras, mesmo inseridas no setor formal, a exemplo da empregada doméstica, ou com frágil relação empregatícia, caso das mulheres trabalhadoras da lavoura, ou ainda as autônomas, como as diaristas, ainda não estão resguardadas pela lei vigente. Logo, embora os planos de retorno ao trabalho possam não influenciar na decisão 
de continuar o aleitamento materno ${ }^{42}$ ou mesmo de mantê-lo exclusivo até esse regresso, a iminência da volta ao trabalho torna inexorável a decisão de incluir precocemente outro tipo de leite no esquema alimentar da criança, constrangendo o sucesso do aleitamento materno complementado e incrementando o tempo mediano da duração do aleitamento misto, conforme indicaram os resultados deste estudo. Salienta-se, ainda, que essa nova lei não garantirá que as mulheres trabalhadoras e suas crianças recebam os benefícios da proteção à amamentação, se falhar o apoio institucional, governamental, familiar e do serviço de saúde.

Sem dúvida, o modo de olhar o mundo pelos grupos populacionais que residem na área rural de vários estados e municípios do país, de diferentes níveis de desenvolvimento socioeconômico, é um fator positivo na preservação de práticas de vida saudáveis e de conservação de estado solidário de viver 31. Desse modo, pode-se entender o resultado desta pesquisa, segundo a qual residir na área rural imprime menor risco de interrupção precoce do aleitamento materno exclusivo e total, em comparação a residir na zona urbana. Esse achado foi similar ao registrado por Assis et al. ${ }^{31}$ e Ramos et al. ${ }^{43}$, para outros grupos de crianças brasileiras.

Projeções recentes indicam que, mantidas as taxas de crescimento do aleitamento materno atuais, seriam necessários cerca de trinta anos para que $90 \%$ das crianças brasileiras estivessem cobertas pelos benefícios da amamentação ao peito, conforme recomendações dos organismos nacionais e internacionais 44 . Essas evidências, mesmo que sombrias, não sustentam que o cenário nacional seja desfavorável para a melhoria da prática do aleitamento materno.

Assim, os resultados da presente investigação, somados àqueles substanciados em estudos bem conduzidos, remetem à necessidade de reflexão sobre a revisão das políticas públicas voltadas para a promoção da prática adequada do aleitamento materno nos dois primeiros anos de vida da criança. Para tanto, deve ser contemplada a relevância dos fatores ambientais, sociodemográficos, antropométricos e biológico-obstétri- cos relacionados a esse evento, contextualizados em um mundo globalizado e em constante mudança, que nem sempre prima pelo desenvolvimento social e humano com base na ampliação dos indicadores do estilo de vida saudável, em especial para garantir a nutrição adequada e saúde das mães e de suas crianças.

Vale destacar que estudos de coorte podem estar sujeitos a limitações impostas por vieses. No entanto, as limitações que poderiam ser registradas neste trabalho são mínimas, uma vez que as perdas de acompanhamento foram inexpressivas e indicativas de que ocorreram de forma aleatória, motivos pelos quais não foram realizadas análises estatísticas para avaliar a comparabilidade entre características das perdas e seguidos até o final do estudo. Acredita-se, também, que o viés de informação foi minimizado, já que o conteúdo do aconselhamento para alimentação saudável nos dois primeiros anos de vida foi padronizado e realizado de forma similar para todas as mães. O material instrucional foi fornecido a cada uma delas e os contatos com a equipe de saúde ocorreram de forma similar entre os grupos.

Outro destaque é o fato de que a informação sobre o desfecho, ou seja, sobre modificações no esquema alimentar da criança ao longo do tempo, possa, em algum momento, ter sido distorcida pelo cuidador da criança e promovido à classificação em categorias de exposição também distorcida. Esse fato pode ocorrer ainda que regras claras tenham sido definidas para a classificação da exposição e do desfecho. Salienta-se que, se isso ocorreu neste estudo, deve ter atingido indistintamente os grupos de exposição diluindo a influência do erro da classificação na medida do desfecho.

Destaca-se ainda que é improvável que este estudo tenha sofrido influência negativa de viés de seleção para participação no estudo, pois todas as crianças nascidas no período pré-estabelecido tiveram a mesma chance de participar. Em adição, os casos de endereços não localizados, admissão hospitalar materna em estágios avançados do trabalho de parto e altas precoces foram situações esporádicas. 


\section{Resumo}

Este estudo objetivou identificar a duração mediana e os fatores associados à interrupção precoce do aleitamento materno. Envolveu uma coorte de nascimento de 531 crianças acompanhadas até os dois anos de idade em dois municípios do Recôncavo da Bahia, Brasil. Utilizaram-se a análise de sobrevivência e o modelo multivariado de Cox. A duração mediana foi de 74,73, 211,25 e 432,63 dias, respectivamente, para o aleitamento materno exclusivo, misto complementado e total. A ausência materna ao pré-natal elevou em 173\% (HR = 2,73; IC95\%: 1,89-3,93) o risco de diminuir a duração do aleitamento materno exclusivo, em 83\% (HR = 1,83; IC95\%: 1,06-3,16) o risco da adoção do aleitamento misto complementado e em $38 \%$ (HR = 1,38; IC95\%: 1,06-1,81) o risco da descontinuidade do aleitamento materno. $O$ trabalho materno fora do domicílio e a área de residência urbana aumentaram o risco para interrupção precoce do aleitamento materno. A ampliação do acesso ao pré-natal e da rede de proteção às mães que trabalham fora do domicilio e àquelas que residem na área urbana poderia aumentar a duração da amamentação no Recôncavo da Bahia.

Aleitamento Materno; Lactação; Nutrição do Lactente; Estudos de Coortes

\section{Colaboradores}

F. Demétrio concebeu o artigo, realizou parte da produção dos dados e participou da redação e revisão de todas as etapas de elaboração do manuscrito. E. J. Pinto participou da análise estatística dos dados e da elaboração do manuscrito. A. M. O. Assis foi responsável pelo planejamento, desenho, captação de recurso, produção dos dados, coordenação da pesquuisa e orientação deste trabalho e contribuiu com a elaboração deste.

\section{Agradecimentos}

Ao CNPq (processo no. 505971/04-6), à Fundação de Amparo à Pesquisa do Estado da Bahia e ao Centro Colaborador em Alimentação e Nutrição Nordeste II/MS, pelo financiamento do trabalho de campo; ao Núcleo de Epidemiologia Nutricional pelo suporte técnicocientífico; às mães e suas crianças, por terem participado do estudo; às prefeituras dos Municípios de Laje e Mutuípe, pelo apoio logístico.

\section{Referências}

1. Spyrides MHC, Struchiner CJ, Barbosa MTS, Kac G. Amamentação e crescimento infantil: um estudo longitudinal em crianças do Rio de Janeiro, Brasil, 1999/2001. Cad Saúde Pública 2005; 21:756-66.

2. The Baby Friendly Initiative. The effectiveness of Baby Friendly accreditation in increasing breastfeeding rates. 2008. http://http://www.babyfriendly. org.uk/items/research_detail.asp?item $=530$ (acessado em 23/Out/2008).

3. Departamento de Ações Programáticas e Estratégicas, Secretaria de Atenção à Saúde, Ministério da Saúde. II Pesquisa de Prevalência de Aleitamento Materno nas Capitais Brasileiras e Distrito Federal. Brasília: Ministério da Saúde; 2009.

4. WHO Collaborative Study Team on the Role of Breastfeeding on the Prevention of Infant Mortality. Effect of breastfeeding on infant and child mortality due to infectious diseases in less developed countries: a pooled analysis. Lancet 2000; 355:451-5.

5. Department of Child and Adolescents Health and Development, World Health Organization. Evidence on the long-term effects breastfeeding: systematic review and meta-analyses. Geneva: World Health Organization; 2007.

6. Javorski M, Scochi CGS, Lima RAG. Os programas nacionais de incentivo ao aleitamento materno: uma análise crítica. Pediatr Mod 1999; 35:30-6.

7. Assis AMO, Barreto ML, Santos LMP, Sampaio LR, Magalhães LP, Prado MS, et al. Condições de vida, saúde e nutrição na infância na cidade de Salvador. Salvador: Bureau, Gráfica e Editora; 2000.

8. Lauer JA, Betran AP, Victora CG, Onis M, Barros AJ. Breastfeeding patterns and exposure to suboptimal breastfeeding among children in developing countries: review and analysis of nationally representative surveys. BMC Med 2004; 2:26.

9. Rosner B. Fundamentals of biostatistics. $4^{\text {th }}$ Ed. New York: Duxbury Press; 1995.

10. World Health Organization. Indicators for assessing infant and young child feeding practices. Part 1 definitions. Washington DC: World Health Organization; 2007.

11. Assis AMO, Gaudenzi EN, Gomes G, Silva RCR, Szarfarc SC, Souza SB. Níveis de hemoglobina, aleitamento materno e regime alimentar no primeiro ano de vida. Rev Saúde Pública 2004; 38:543-51.

12. Lohman TG, Roche AF, Martorell R. Anthropometric standardization reference manual. Champaign: Human Kinetics Books; 1988.

13. Oliveira VA, Assis AMO, Pinheiro SMC, Barreto ML. Determinantes dos déficits ponderal e de crescimento linear em menores de dois anos. Rev Saúde Pública 2006; 40:874-82. 
14. Ministério da Saúde/Organização Pan-Americana da Saúde. Guia alimentar para crianças menores de 2 anos. Brasília: Ministério da Saúde; 2002. (Série A. Normas e Manuais Técnicos, 107).

15. Kaplan EL, Meier P. Nonparametric estimation from incomplete observations. J Am Stat Assoc 1958; 53:457-81.

16. Colósimo EA, Giolo SR. Análise de sobrevivência aplicada. São Paulo: Edgard Blucher; 2006.

17. Mantel N. Evaluation of survival data and two new rank order statistics arising in its consideration. Cancer Chemother Rep 1966; 6:163-8.

18. Ministério da Saúde. Pesquisa de prevalência do aleitamento materno nas capitais e no Distrito Federal: relatório. Brasília: Ministério da Saúde; 2001.

19. Ministério da Saúde. Pesquisa Nacional de Demografia e Saúde da Criança e da Mulher PNDS 2006: dimensões do processo reprodutivo e da saúde da criança. Brasília: Ministério da Saúde; 2006.

20. Bueno MB, Souza JMP, Souza SB, Paz SMRS, Gimeno SGA, Siqueira AAF. Riscos associados ao processo de desmame entre crianças nascidas em hospital universitário de São Paulo, entre 1998 e 1999: estudo de coorte prospectivo do primeiro ano de vida. Cad Saúde Pública 2003; 19:1453-60.

21. Victora CG, Matijasevich A, Santos IS, Barros AJD, Horta BL, Barros FC. Breastfeeding and feeding patterns in three birth cohorts in southern Brazil: trends and differentials. Cad Saúde Pública 2008; 24 Suppl 3:S409-16.

22. Lira PIC, Lima MC, Silva GAP, Romani SAM, Eickmann SH, Alessio MLM, et al. Saúde e nutrição de crianças de áreas urbanas da Zona da Mata Meridional de Pernambuco: resultados preliminares de um estudo de coorte. Rev Bras Saúde Matern Infant 2003; 3:463-72.

23. Damião JJ. Influência da escolaridade e do trabalho maternos no aleitamento materno exclusivo. Rev Bras Epidemiol 2008; 11:442-52.

24. Vasconcelos MGL, Lira PIC, Lima MC. Duração e fatores associados ao aleitamento materno em crianças menores de 24 meses de idade no estado de Pernambuco. Rev Bras Saúde Matern Infant 2006; 6:99-105.

25. Oliveira LPM, Assis AMO, Gomes GSS, Prado MS, Barreto ML. Duração do aleitamento materno, regime alimentar e fatores associados segundo condições de vida em Salvador, Bahia, Brasil. Cad Saúde Pública 2005; 21:1519-30.

26. Medeiros JS, Rivera MAA, Benigna MJC, Cardoso MAA, Costa MJC. Estudo caso-controle sobre exposição precoce ao leite de vaca e ocorrência de Diabetes Mellitus tipo 1 em Campina Grande, Paraíba. Rev Bras Saúde Matern Infant 2003; 3:27180.

27. Mendes ACR, Valença AMG, Lima CCM. Associação entre aleitamento, hábitos de sucção não-nutritivos e maloclusões em crianças de 3 a 5 anos. Cienc Odontol Bras 2008; 11:67-75.

28. Niquini RP, Bittencourt SA, Lacerda EMA, Leal MC. Fatores associados à introdução precoce de leite artificial, município do Rio de Janeiro, 2007. Rev Bras Epidemiol 2009; 12:446-57.
29. Assis AMO, Monteiro MC, Santana MLP, Santos NS Diagnóstico de saúde e nutrição da população de Mutuípe-BA. Salvador: Editora UFBA; 2002. (Série UFBA em Campo - Estudos e Debates).

30. Rea MF. Reflexões sobre a amamentação no Brasil: de como passamos a 10 meses de duração. Cad Saúde Pública 2003; 19 Suppl 1:37-45.

31. Assis AMO, Prado MS, Freitas MCS, Silva RCR, Ramos LB, Machado AD. Prática do aleitamento materno em comunidades rurais do semi-árido baiano. Rev Saúde Pública 1994; 28:380-4.

32. Chaves RG, Lamounier JA, César CC. Factors associated with duration of breastfeeding. J Pediatr 2007; 83:241-6.

33. Scott JA, Binns CW, Oddy WH, Graham KI. Predictors of breastfeeding duration: evidence from a cohort study. Pediatrics 2006; 117:646-55.

34. Sandre-Pereira G, Colares LGT, Carmo MGT, Soares EA. Conhecimentos maternos sobre amamentação entre puérperas inscritas em programa de pré-natal. Cad Saúde Pública 2000; 16:457-66.

35. Baptista GH, Andrade AHHKG, Giolo SR. Fatores associados à duração do aleitamento materno em crianças de famílias de baixa renda da região sul da cidade de Curitiba, Paraná, Brasil. Cad Saúde Pública 2009; 25:596-604.

36. Departamento de Atenção Básica, Secretaria de Atenção à Saúde, Ministério da Saúde. Dez passos para uma alimentação saudável. Guia alimentar para menores de dois anos. Um guia para o profissional da saúde na atenção básica. Brasília: Ministério da Saúde; 2010.

37. Schwartz K, D'Arcy HJS, Gillespie B, Bobo J, Longeway M, Foxman B. Factors associated with weaning in the first 3 months postpartum. J Fam Pract 2002; 51:439-44.

38. Dearden K, Altaye IM, Oliva MSJ, Morrow AL, Burkhalter BR. Determinants of optimal breast-feeding in peri-urban Guatemala City, Guatemala. Rev Panam Salud Pública 2002; 12:185-92.

39. Fein SB, Mandal B, Roe BE. Success of strategies for combining employment and breastfeeding. Pediatrics 2008; 122 Suppl 2:S56-62.

40. Taddei JAAC, Westphal MF, Venâncio S, Bogus C, Souza S. Breastfeeding training for health professionals and resultant changes in breastfeeding duration. São Paulo Med J 2000; 118:185-91.

41. Brasil. Lei no. 11.770/08. Cria o Programa Empresa Cidadã, destinado à prorrogação da licença-maternidade mediante concessão de incentivo fiscal, e altera a Lei no 8.122 de 24 de julho de 1991. Diário Oficial da União 2008; 9 set.

42. Ciaccia MCC, Ramos JLA, Issler H. Amamentação e trabalho da mulher: como conciliar. Rev Paul Pediatr 2003; 21:83-8.

43. Ramos CV, Almeida JAG, Alberto NSMC, Teles JBM, Saldiva SRDM. Diagnóstico da situação do aleitamento materno no Estado do Piauí, Brasil. Cad de Saúde Pública 2008; 24:1753-62.

44. Parizoto GM, Parada CM, Venancio SI, Carvalhaes MA. Trends and patterns of exclusive breastfeeding for under-6-month-old children. J Pediatr 2009; 85:201-8.

Recebido em 30/Mai/2011

Versão final reapresentada em 28/Nov/2011 Aprovado em 01/Dez/2011 
Demétrio F, Pinto EJ, Assis AMO. Fatores associados à interrupção precoce do aleitamento materno: um estudo de coorte de nascimento em dois municípios do Recôncavo da Bahia, Brasil. Cad Saúde Pública 2012; 28(4):641-654.

A revista foi informada sobre um erro no abstract. O abstract correto é:

The journal has been informed about an error in the abstract. The correct abstract is:

\begin{abstract}
This study aimed to identify the median duration of breastfeeding and associated factors in a cohort of 531 infants in two municipalities in the Recôncavo region, Bahia State, Brazil. Breastfeeding duration was estimated by survival analysis and its associations by the Cox multivariate model. Median duration of exclusive breastfeeding, mixed breastfeeding with complementary feeding, and total breastfeeding was 74.73, 211.25, and 432.63 days, respectively. Lack of prenatal care increased the risk of shortening exclusive breastfeeding by 173\% (HR = 2.73; 95\%CI: 1.89-3.93), of adopting mixed breastfeeding with complementary feeding by 83\% (HR = 1.83; 95\%CI: 1.06-3.16), and of discontinuing breastfeeding entirely by $38 \%$ (HR $=1.38 ; 95 \% C I: 1.06-1.81)$. Both maternal employment and residence in an urban area increased the risk of early breastfeeding cessation. Expansion of access to prenatal care and the safety network for employed mothers living in urban areas could increase breastfeeding duration in the target region.
\end{abstract}

